\title{
MYASTHENIA GRAVIS.
}

\author{
By J. SHAFAR, M.D., D.P.H. \\ (Medical Registrar, West End Hospital for Nervous Diseases, and Willesden General \\ Hospital.)
}

The syndrome of myasthenia gravis was first described by Willis in the seventeenth century, but Erb in I878 gave the first detailed account of the condition as a clinical entity. It is characterized by an abnormal fatiguability of the muscular system, resulting in weakness, which in the early stages is relieved by rest, but which is eventually succeeded by permanent paralysis.

The usual age of onset is in the third decade, but it is probably much commoner in childhood than is generally recognized, while at the other extreme records are present of patients in whom the earliest symptoms appeared after the age of sixty. There is a somewhat greater incidence in the female sex. No hereditary or familial tendencies have been noted.

The ætiology of the condition is by no means clear, but certain recent advancements in our knowledge have thrown some light on the possible nature of the abnormality. Acetylcholine is the chemical substance which is liberated at the myoneural junction after the stimulation of a motor nerve to skeletal muscle. It has been proved that acetylcholine is the transmitter of the excitory process at the myoneural junction. The pharmacological action of this mediator is threefold. Firstly, there is a local dilator effect on certain blood vessels; secondly, a muscarine action which closely mimics the results produced by stimulation of the parasympathetic division of the autonomic nervous system; and lastly, a nicotine effect, which is dependent on the stimulation of the autonomic ganglia.

The extraordinary transient nature of acetylcholine has rendered it unsatisfactory for therapeutic purposes, although other more stable derivatives have been employed with some success. A specific enzyme (choline esterase) which is present in all tissues and in the blood, is responsible for its rapid destruction in the body. This destruction is effected by the conversion of acetylcholine into choline, by a process of hydrolysis, the latter substance being many times less active than its acetyl ester.

Fraser (1938) summarises the prevalent theories of myasthenia gravis, as interpreted in terms of disturbance of acetylcholine transmission. A deficiency may arise from a defective production or an excessive destruction by choline esterase. The other alternative offered is that a curare-like substance is present in the blood of subjects of myasthenia gravis, which antagonises the action of acetylcholine and so reduces its activity. McGeorge (I937) estimated the choline esterase content of the serum of normal individuals and of cases of myasthenia gravis and found that a marked degree of variability existed and that the serum level in this malady was lower than the average normal values. Since the essential factor is the esterase activity at the myoneural junction, the presence of a normal serum esterase cannot be accepted as proof of normality of esterase function. In another series of investigations Fraser, McGeorge and Murphy (I937) concluded that some defect of acetylcholine production was probably present in myasthenia gravis. Walker (I938) demonstrated a case of myasthenia gravis, in whom after exercise of an arm whose circulation had been cut off by a sphygmomanometer, the effect of release of the constricting band, was to produce an increase of weakness in the muscles generally. This experiment appears to lend support to the theory that some substance is liberated into the circulation to produce an antagonising action on acetylcholine. 
For many years, it has been observed that an anomaly of the thymus gland is frequently present in cases of myasthenia gravis. Norris (I936) estimates the incidence at fifty per cent., and states that the usual changes are those of hyperplasia or a benign thymoma. Occasionally, a malignant neoplasm of the thymus is present. The significance of these thymic changes is not yet apparent, and the experiments of those workers who have been able to demonstrate muscular weakness in animals, following injections of thymic extracts requires repetition and confirmation (Adler, I937).

Another ductless gland has been implicated in the ætiological panel. Exophthalmic goitre is a disease in which muscular weakness is often a prominent symptom, and not infrequently the myasthenia is localised in certain muscle groups : moreover, myasthenia gravis and thyrotoxicosis not infrequently co-exist. They present several features in common. In both there may be generalised hyperplasia of lymphoid tissue, lymphorrhages in the muscles and a lymphocytosis in the blood. The association of exophthalmic goitre with familial periodic paralysis is also well known, and it would appear that the thyroid disorder predisposes in susceptible individuals to the development of the muscular disorder (Nevin, I938).

Pathological examinations in the vast majority of cases reveal no abnormalities in the central nervous system; the occurrence of minor cellular changes in a few cases has not been held to be causally related. Lymphorrhages are accumulations of lymphocytes found chiefly in the muscles but also seen in the internal organs; the ocular muscles are the commonest site of their occurrence. The changes in the thymus and thyroid glands have been already mentioned. No alteration of the affected muscle is observed, apart from those which have developed as a secondary state to a long period of paralysis.

The metabolic disturbances met with in some cases are not characteristic of the disease. A diminished sugar tolerance, indicated by a high blood sugar curve and slight glycosuria after the ingestion of a test dose of glucose may be encountered. Adams, Powers and Boothby (I936) found the blood magnesium normal in six cases and no abnormality in the other inorganic or nitrogenous constituents of the blood. Nevin (I934) concluded that the chemical processes which accompany activity in myasthenic muscles differed in no significant manner from those processes which are characteristic of healthy muscle. As in all muscle disturbances in which a decrease of muscle activity or contraction is present, creatinuria has been noted.

The onset of the affliction is usually gradual, but in a minority of instances it has been abrupt and the course very rapid. Characteristically, the condition is subject to remissions and relapses, both of varying duration. The latter have frequently followed on an acute infectious disease, though not upon any particular infection. As far as is known at present, no external factors have any influence on the production of a remission.

The ocular muscles are usually among the first to be involved and in practically every case are implicated at some stage in the course of the affection. Those muscles which derive their nerve supply from the bulbar nuclei, are also among the earliest attacked. Diplopia, ptosis, difficulty in holding up the neck and inability to continue the act of mastication during a meal are frequently presenting symptoms. The ophthalmoplegia may involve any of the extra-ocular muscles; difficulty in convergence of the eyes is also encountered, while the ptosis of the upper eyelid may be uni- or bilateral. A peculiar facies, produced by weakness of the facial musculature with consequent smoothing out of the wrinkles of the face, 
is recognisable. The face is more or less expressionless and when the patient attempts to smile, a snarling nasal smile results-the so-called " myasthenic smile" - since the only muscles which are active in the movement are the naso-labial elevators. Swallowing and chewing become difficult and tongue movements are easily fatigued. Speech is thin and high pitched, and after a varying period its audibility decreases until the whispered voice sounds only can be expressed (Grinker, I937). The fatigue of the vocal cords can be visualised by the laryngoscope. The musculature of the limbs tend to show some involvement but this is as a rule of less marked degree. Laughing and crying which produce excessive activity of the respiratory muscles, may produce dangerous dyspnœa should this group be fatiguable.

The essential feature of this muscular weakness, is that in the earliest stages the weakness passes off after a period of rest and perhaps only appears towards the end of the day, the patient arising in the morning free from symptoms. Gradually, the amount of exercise necessary to produce the fatigue becomes less and less and longer rest is required to afford relief, until eventually it proves ineffective and a permanent paralysis results. During a remission, however, the patient may show no evidence of excessive fatiguability of any of his muscles. The course of the disease in the majority of cases shows a slow advancement to a fatal issue, but in a smaller series the condition may terminate after a short existence of weeks or months. Death usually results from respiratory failure or an aspiration pneumonia, but these patients are liable to sudden death, which is evidently cardiac in origin.

Examination of the various reflexes usually reveals no abnormal findings, but occasionally they may be diminished or lost as a result of the extreme muscular weakness. Sensation is normal and co-ordination unaffected. There are no mentalo changes or involvement of the sphincters of the bladder or rectum, but peculiar nystagmoid movements of the eyes are commonly encountered and while the response of the pupil to light is in the majority of cases brisk, it is in a few instances sluggish and even absent.

The myasthenic reaction of Jolly, is not diagnostic of myasthenia gravis, but is also found in muscles which are recovering from paralysis of the lower motor neurone type. It consists of a gradual diminution of the muscular contraction under the influence of a constant faradic current, until actual flaccidity occurs. After a moment's rest the part recovers, only to fatigue rapidly again.

The treatment of the condition has improved but is still disappointing. No effect on the actual progress is produced, nor has any mode of therapy been able to influence the incidence of a remission. Several drugs have been introduced which have a palliative action and which are of great value from this aspect. Nevertheless rest must remain the most important factor and must be graded according to the severity of the paralysis and the areas affected.

Edgeworth in I930, herself a subject of the malady, noticed the beneficial effects of ephedrine and this has been confirmed by other writers (McAlpine, I934). It is customary to give half a grain once or twice daily.

Glycine has been claimed to be of value (Boothby, 1932) but there appears to be no theoretical rationale for its use and the general concensus of opinion among neurologists is that it has no place in the therapeutic armamentarium.

Walker (I935) used eserine but found that although an increase in muscular power was obtained, the drug had toxic complications in an effective dosage, which rendered it unsuitable for treatment. Using a synthetic preparation known as 
prostigmin, she stated that the action of this medicament was more powerful than that of eserine and the toxicity less. These observations have been confirmed by others. Prostigmin belongs to that class of drugs known as parasympathetic agents ; pharmacologically it differs from eserine in having a more pronounced effect on peristalsis, but relatively little action on the heart. Toxic symptoms are encountered, however, and consist of nausea, perspiration, dizziness, muscle cramps and gastro-intestinal upsets. These disturbances last for a short period only, usually I5 to 30 minutes; the administration of belladonna or atropine abolishes these untoward by-effects. Prostigmin affords a temporary relief, but has no influence on the ultimate course or issue of the malady. Nevertheless, it allows the patient to lead a more varied existence, improves the general health and enables him to eat more adequate meals. The drug may be administered by intramuscular or subcutaneous injection, but recently it has been found that the oral route is effective in myasthenia gravis. The beneficial effects produced on the muscular weakness are dramatic, but of temporary duration; the effect becomes manifest ten minutes after injection and within half an hour if taken by mouth, but passes off in three to eight hours. The dosage must vary with the individual case. The average dose is 45 to $60 \mathrm{mgs}$. per day orally. Injections are usually reserved for some special occasions such as a visit to the theatre. Some patients appear to acquire some tolerance to the drug, while another important feature is that mental depression and increased muscular fatigue may occur after the initial improvement. The spacing of the doses must depend on the periodicity of the myasthenia which in the early cases is only apparent towards the end of the day, but which in others may be present from early morning.

Prostigmin is also of great value in diagnosis. For this purpose it is given by injection and the improvement, if any, on the myasthenia is noted. One must first determine the best objective symptoms for evidence of improvement, give the injection at a time when these symptoms are most marked, and observe the patient at ten minute intervals for an hour (Schwab and Veits, I938). An intramuscular injection of 3 c.c. prostigmin to which has been added I/IOO gr. atropine is administered for the diagnostic test. An important advance has been made by Harvey and Whitehill (I937) who utilise quinine as well as prostigmin in the diagnosis of myasthenia gravis. Quinine increases the myasthenic symptoms and the improvement following an injection of prostigmin is made more apparent.

There have been some investigations into the value of acetylcholine and its derivatives in combination with prostigmin, and although the reports are encouraging, the work is of too recent origin to draw definite conclusions.

Potassium chloride in doses of two grammes given four to six times a day has been found of value as an adjuvant to prostigmin (Laurent and Walker, I935). Toxic effects have been reported and the drug must be used with caution.

\section{Case Reports.}

Case 1. The patient, a girl of 19 years of age, was first seen in March, 1937. She complained of stiffness and weakness of the jaws during meals which necessitated temporary cessation of the act of mastication, but which was relieved after a few minutes' rest. These symptoms first appeared in October, 1936 and have persisted since. Several months later she developed periodic diplopia and dysphagia with regurgitation of fluids through the nose. These features were noticeable in the evening. The tongue movements became clumsy and her voice indistinct, when she was tired. Resting produced a return to normal. The past and family history contained no relevant 
facts. Examination of the various stems revealed no abnormalities beyond weakness of the affected group of muscles during an attack of myasthenia.

The patient was placed on $15 \mathrm{mg}$. prostigmin orally thrice daily with a favourable $\overrightarrow{\bar{N}}$ response. The addition of potassium chloride was tried, in an attempt to enhance the $\vec{\sigma}$ action of prostigmin, but the patient reacted after several doses with a sudden collapse, sweating and pallor; the pulse was almost imperceptible. Ephedrine gr. $\frac{1}{2}$ twice daily was substituted and this proved of value in controlling the troublesome salivation. Prostigmin therapy has been continued up to the present date-that is, over a period of $\mathrm{n}$ two years. No toxic symptoms have been noted, but the drug has become léss effective in controlling the myasthenia. Whether this is due to an acquired tolerance or increase in severity of the condition, is difficult to determine, but the course of the malady has $\vec{\omega}$ shown no signs of remission or of severe retrogression. The dose of prostigmin was $\frac{0}{0}$ increased to four $15 \mathrm{mg}$. tablets a day, but the patient complained that following this addition, she felt dull and depressed in the morning and the stiffness and weakness of the muscles were greater after the initial improvement following the administration of prostigmin. She herself reduced the dosage to $45 \mathrm{mg}$. daily, taking one tablet before breakfast, lunch and dinner.

Case 2. The patient, a woman of 32 years of age, complained of drooping of the eyelids of 18 months' duration. Ptosis appeared in the evening and was occasionally accompanied by diplopia and a weakness of the voice. When she was first seen in December, 1938 examination of various systems failed to demonstrate any abnormal physical signs, but towards the end of the day, the ocular manifestations became $\stackrel{\Phi}{\mathscr{Q}}$ evident.

There had been no serious illness in the past and no family incidence was present The cerebrospinal fluid showed no abnormal findings.

Prostigmin was prescribed in February, 1939 in doses of $15 \mathrm{mg}$. thrice daily and the improvement in the muscular weakness has so far been maintained.

REFERENCES.

ADAMS, M., POWERS, M. H. and BOOTHBY, W. M. (1936), Ann. Int. Med., 9, 823.

ADLER, H.' (1937), Arch. Klin. Chir., 187, 528.

BOOTHBY, W. H. (1932), Proc. Mayo. Clin., 7, 557.

EDGEWORTH, H. (1930), J.A.M.A., 94, 1136

FRASER, F. R. (1938), Brit. Med. Jourm 1349

FRASER, F. R. McGEORGE, M. and MÜRPHY, G. E. (1937), Clin. Sci., 3, 77

FRASER, F. R., MCGEORGE, M. and MURPHY, G. E. (1937), Clin. Sci., 3, 77,

GRINKER, R. (1937), "Neurology" (2nd Edition), Bailière, Tindall \& Cox, Londo

LAURENT, L. P. E. and WALKER, M. B. (1935), Lancet, 1, 1434.

MCALPINE, D. (1934), Lancet, 1, 180.

NEVIN, S. (1934), Brain, 57, 239.

NEVIN, S. (1938), J. Neurol., 1, 120.

NORRIS, E. H. (1936), Am. J. Cancer, 27, 421.

SCHWAB, R. S, and VEITS, H. R. (1938), New Eng. J. Med., 219, 226.

WALKER, M. B. (1935), Lancet, $2,47$.

WALKER, M. B. (1938), Proc. Roy. Soc. Med., 31, 722. 